\title{
Pesquisa
}

\section{Conhecimento dos Acadêmicos de Odontologia Sobre a Dor Pós- Operatória em Endodontia}

\author{
Knowledge of Dental Students on Postoperative Pain in Endodontics
}

Flaviana Lais Pereira Dos Santos ${ }^{1}$; Cynára Liane Jales Ataide De Melo1; Fernanda Yasmin Teixeira Rodrigues2; Magnólia Fernandes Ferreira2; Victorya De Lima Spinellis Do Nascimento'; Consuelo Fernanda Macedo Souza ${ }^{3}$

${ }^{1}$ Graduanda do Centro Universitário de João Pessoa- UNIPÊ- João Pessoa, Paraíba, Brasil. ${ }^{2}$ Cirurgiã-Dentista.

3Professora adjunta do Centro Universitário de João Pessoa- UNIPÊ- João Pessoa, Paraíba, Brasil.

Autor e endereço para correspondência:

Consuelo Fernanda Macedo Souza - Rua Golfo de Sião 77, Ponta de Campina, Cabedelo-PB, 58.310-000. E-mail: consuelofernanda79@hotmail.com.

\begin{abstract}
Resumo
Introdução: Existem graus de sensação dolorosa antes, durante e após o tratamento endodôntico. No entanto um dos principais problemas do estudo da dor é a dificuldade de sua avaliação, pois há uma variação do limiar e percepção da dor. Objetivo: avaliar o conhecimento dos acadêmicos de odontologia da Clínica Integrada do Centro Universitário de João Pessoa (UNIPÊ) acerca da dor pós-operatória endodôntica. Metodologia: Esse estudo é observacional, transversal, quantitativo, descritivo e exploratório. Foram aplicados 114 questionários em um universo de 122 alunos, e obedecidas às recomendações da Res. 466/12-CNS/MS. Os dados coletados foram analisados por meio de estatística descritiva. Resultados: Os acadêmicos (44,7\%) que acreditam que a substância química auxiliar tem papel importante na Dor Pós Operatória (DPO), (29,4\%) confiam ser a solução de Milton $1 \%$ a maior responsável pela DPO, seguida pela clorexidina $2 \%(21,8 \%)$. Com relação ao tipo de PQM empregado no tratamento endodôntico, (73,3\%) consideram que esse é um fator que pode ser responsável pela DPO, sendo a técnica ápice-coroa (46\%) a maior responsável pelo surgimento de DPO e as técnicas mecanizadas $(24,1 \%)$ as que geram menos dor após o procedimento. Quanto ao tipo de medicação sistêmica utilizada por quadro clínico percebeu-se que $(15,1 \%)$ prescreveriam analgésicos e $(11 \%)$ antiinflamatórios para um quadro de pulpite, $(6,8 \%)$ fariam a associação medicamentosa para o flare up, o quadro de periodontite aguda seria tratado basicamente com antiinflamatório $(7,8 \%)$ e antibiótico (10\%), já a periodontite crônica com antibioticoterapia (10\%). Conclusão: conclui-se que os estudantes apresentam deficiência nos conhecimentos a respeito da DPO e principalmente sobre prescrição medicamentosa.
\end{abstract}

Descritores: Endodontia. Forame apical. Manejo da dor. 


\begin{abstract}
Introduction: There are degrees of painful sensation before, during and after endodontic treatment. However one of the main problems of the study of pain is the difficulty of its evaluation, because there is a variation of the threshold and perception of pain. Objective: to evaluate the knowledge of dentists at the Integrated Clinic of the University Center of João Pessoa (UNIPÊ) about postoperative endodontic pain. Methodology: This study is observational, transversal, quantitative, descriptive and exploratory. 114 questionnaires were applied in a universe of 122 students, and obeyed the recommendations of Res. 466/12-CNS / MS. The data collected were analyzed using descriptive statistics. Results: The authors (44.7\%) who believe that the auxiliary chemical plays an important role in postoperative pain (DPO), $(29.4 \%)$ believe that Milton's solution is $1 \%$ the most responsible for DPO, followed by chlorhexidine $2 \%$ (21.8\%). Regarding the type of PQM used in endodontic treatment, (73.3\%) consider that this is a factor that may be responsible for POD, and the apex-crown technique $(46 \%)$ is the most responsible for the appearance of POD and the techniques mechanized (24.1\%) those that generate less pain after the procedure. As to the type of systemic medication used by clinical status, it was observed that $(15.1 \%)$ would prescribe analgesics and (11\%) anti-inflammatories for pulpitis, $(6.8 \%)$ would make the drug association for flare up, $(7,8 \%)$ and antibiotic (10\%), and chronic periodontitis with antibiotic therapy (10\%). Conclusion: it is concluded that the students are deficient in the knowledge about the DPO and mainly about medication prescription.
\end{abstract}

Key-words: Endodontics. Apical foramen. Pain management.

\title{
Introdução
}

A dor pós-operatória (DPO) é uma situação que pode desenvolver-se em qualquer período do tratamento, seja no início ou após sua finalização (flare-up). O desenvolvimento da DPO na endodontia se dá devido a inflamação aguda estimulada durante o procedimento nos tecidos perirradiculares ${ }^{1}$. Além disso, a dor pode ocorrer devido a anestesia ineficaz, devido ao número de sessões, por causa da chance de recontaminação, por traumas na instrumentação e de uma extrusão periapical que é causada de forma fisiológica durante o tratamento no momento da limpeza, pela ocorrência de uma medição incorreta que leva a uma sobre-instrumentação podendo gerar dor pós-operatória; por altas concentrações das substâncias químicas auxiliares, pois causam irritações e inflamações teciduais, entretanto, estudos indicam que a DPO causada pelo hipoclorito a 5,25\% regride bastante após 7 dias da intervenção ${ }^{2-4}$.

O flare-up endodôntico é definido como continuação de dor pré-tratamento e/ou aparecimento de dor e edema após o início do tratamento endodôntico, ou seja, em muitos casos o paciente apresenta um quadro crônico sem presença de dor e após o início do procedimento pode haver 
aparecimento de DPO, fazendo com que ele volte ao consultório para realizar algum tratamento para aliviar os sintomas ${ }^{5}$.

Outro fator importante é a dor ligada à percussão e sensibilidade, comumente procede da reação a estímulos nocivos como as toxinas bacterianas. Pelo fato da terapia endodôntica não eliminar de imediato a inflamação pré-existente no periápice, a dor que já acometia o paciente antes do procedimento poderá continuar mesmo após a realização do tratamento endodôntico, sendo considerada ainda um quadro de $\mathrm{DPO}^{6}$.

Além disso, a sobreinstrumentação dos canais radiculares é um dos fatores de mais destaque na causa de processo inflamatório, uma vez que pode levar um grande número de irritantes para a o ápice, como por exemplo, microorganismos, soluções irrigadoras e medicações introduzidas no interior do canal. Portanto, a presença desses irritantes nos tecidos perirradiculares estimulam a liberação de mediadores inflamatórios que estimulam ou sensibilizam as terminações nervosas livres. Os estímulos de ordem física causados pelos instrumentos endodônticos ativam diretamente os receptores da dor por um processo bioquímico que envolve a entrada de sódio no interior dos mesmos, provocando a dor aguda de caráter inflamatório. Consequentemente pode-se dizer que a dor é quase sempre diretamente proporcional ao grau de estimulação das terminações nervosas livres do local2.

Frente a DPO, deve-se realizar uma anestesia eficaz com técnicas eficientes durante o tratamento. Seguida de um controle farmacológico, através da prescrição de analgésicos antes e após o tratamento com a finalidade de controlar a DPO. Em caso de prescrição dos antimicrobianos deve levar em consideração o curso e a gravidade da infecção, além da história do paciente. É importante lembrar que deve haver uma análise da situação para usar farmacológicos apenas em casos necessários, como por exemplo, quando existe desconforto ou possível inflamação, podendo ser usado um analgésico ou um anti-inflamatório não esteroidal, seguindo sempre a dosagem adequada. Neste quesito, os principais fármacos utilizados são paracetamol como analgésico e ibuprofeno como anti-inflamatório, porém em casos específicos como gravidez e alteração/doença sistêmica é indicado analisar qual o melhor fármaco ${ }^{7,8}$.

Além disso, alguns pacientes apresentam medo, ansiedade e nervosismo frente ao tratamento endodôntico ou até mesmo diante do dentista. A primeira conduta deve ser acalmar o paciente verbalmente. No entanto, quando a conduta verbal não funciona pode-se abrir mão do uso de farmacológicos específicos para tal situação, os chamados ansiolíticos. Estes medicamentos também estão indicados em casos de procedimento mais invasivo, ou em pacientes cardiopatas e doenças neurológicas convulsivas ${ }^{9}$. 
Devido à variedade de situações clínicas possíveis referentes a dor durante o tratamento endodôntico, o objetivo principal deste estudo foi avaliar o conhecimento dos acadêmicos de odontologia da Clínica Integrada do UNIPÊ acerca da dor pós-operatória endodôntica.

\section{Metodologia}

O presente estudo foi realizado na Clínica Escola de Odontologia do UNIPÊ, João PessoaPB; é do tipo observacional, transversal, quantitativo, descritivo e exploratório. $O$ universo da pesquisa foi composto pelos acadêmicos regularmente matriculados nas Clínicas Integradas I e II do curso de Odontologia do UNIPÊ, totalizando 122 alunos. E a amostra foi censitária e por conveniência, sendo composta por 114 alunos. O critério de inclusão nessa pesquisa foram todos os alunos que estavam regularmente matriculados nas Clínicas Integradas I e II. E o de inclusão os alunos que ainda não haviam concluído as disciplinas de Endodontia I e II e que não assinaram o termo de consentimento livre esclarecido (TCLE).

Após a aprovada no Comitê de Ética em Pesquisa do UNIPÊ na 46를 Reunião extraordinária realizada em 07 de julho de 2016, CAAE: 56950716.0.0000.5176, sob protocolo do CEP: $\mathrm{n}^{\circ}$ 187/2016 e Parecer Consubstanciado: 1.663731. O estudo seguiu a norma que regulamenta a pesquisa envolvendo seres humanos no Brasil - Resolução 466/12-CNS/MS (BRASIL, 2012) 21 que aceitaram a participar. A pesquisa assegurou a privacidade aos sujeitos quanto aos dados confidenciais envolvidos na mesma. Foram aplicados questionários estruturados cuja finalidade foi avaliar o conhecimento dos alunos acerca do tema de estudo. Esses questionários foram compostos por dezesseis questões objetivas, que foram respondidas pelos estudantes na clínica, antes do início dos atendimentos, onde os participantes tiveram todo tempo que julgaram necessário para responder. Foram selecionados aleatoriamente $10 \%$ dos questionários respondidos para que fossem aplicados novamente a fim de avaliar a confiabilidade da ferramenta. Os questionários foram aplicados sempre pela mesma pesquisadora.

Os dados foram tabulados no programa estatístico SPSS (17.0). A análise estatística seguiu uma análise descritiva dos dados para verificação dos objetivos. Foram utilizados testes para verificar as relações entre variáveis e a apresentação foi feita na forma de frequências por análise inferencial pelo teste do Qui-quadrado. O nível de significância utilizado foi de 5\%. 


\section{Resultados}

Foram aplicados 114 questionários em um universo de 122 alunos. Cerca de 64\% dos alunos mostraram ter conhecimento a respeito da ocorrência de DPO e apenas $18,4 \%$ dos alunos atenderam o paciente com DPO, como mostram as Figuras 1 e 2.

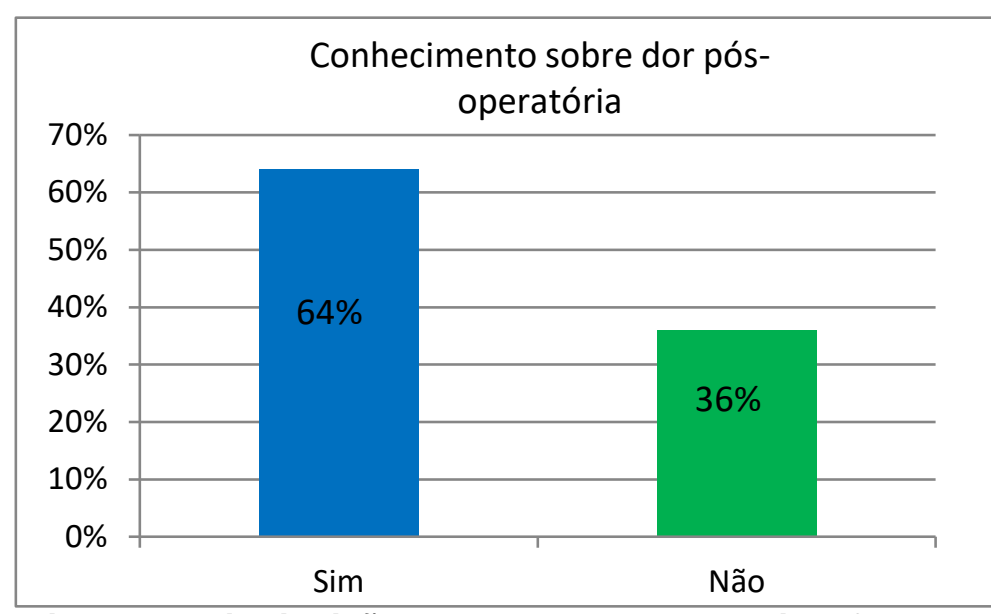

Figura 1 - Distribuição dos valores percentuais referente ao conhecimento sobre a dor pós-operatória. João

Pessoa/PB, 2016.

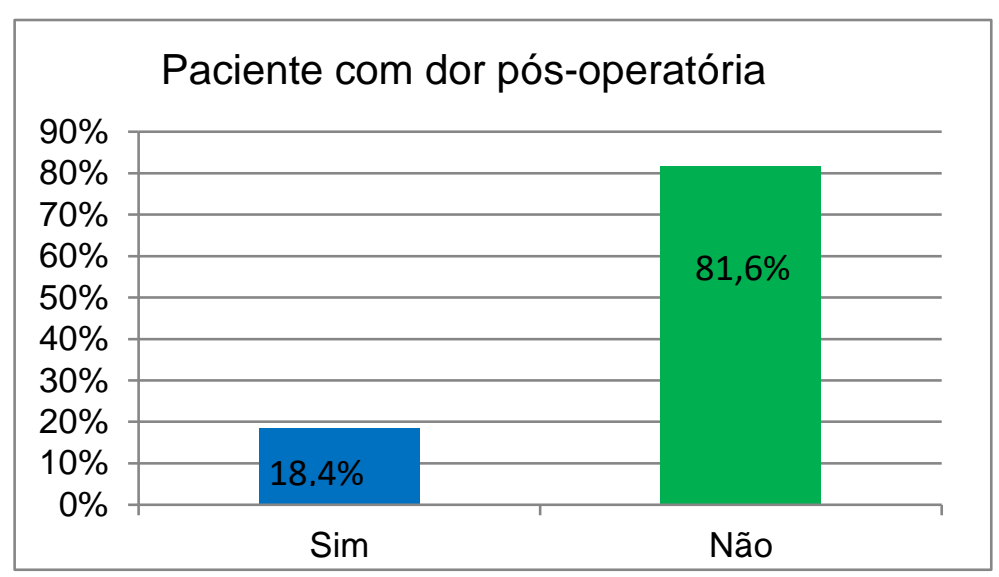

Figura 2 - Porcentagem de atendimentos a paciente com dor pós-operatória. João Pessoa/PB, 2016.

Em relação aos possíveis fatores causadores de DPO, (55,3\%) dos alunos acreditam que o tipo de substância química utilizada no tratamento não tem relação com DPO. Os acadêmicos $(44,7 \%)$ que acreditam que a substância química auxiliar tem papel importante na DPO, $(29,4 \%)$ 
confiam ser a solução de Milton $1 \%$ a maior responsável pela DPO seguida pela clorexidina $2 \%$ $(21,8 \%)$, conforme Figura 3.

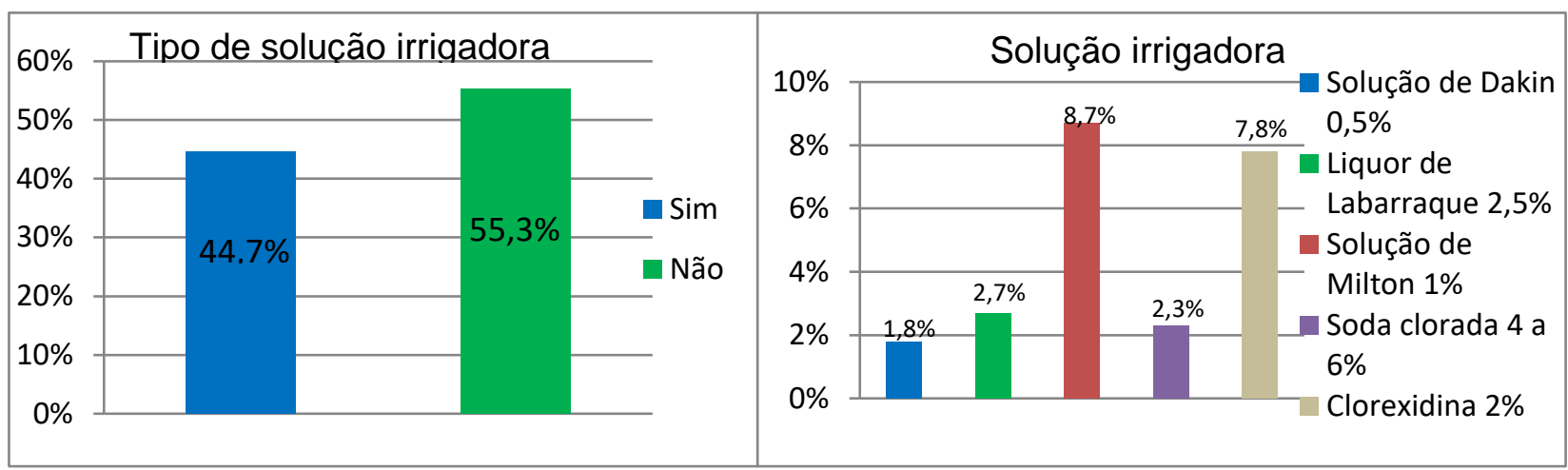

Figura 3 - Relação da substância química irrigadora com a dor pós-operatória. João Pessoa/PB, 2016.

Na questão relacionada ao tipo de PQM utilizado, 76,3\% consideram que esse é um fator que pode ser responsável pela DPO. Sendo a técnica ápice-coroa (46\%) a maior responsável pelo surgimento de DPO e as técnicas mecanizadas $(24,1 \%)$ as que geram menos dor após o procedimento, conforme Figura 4.

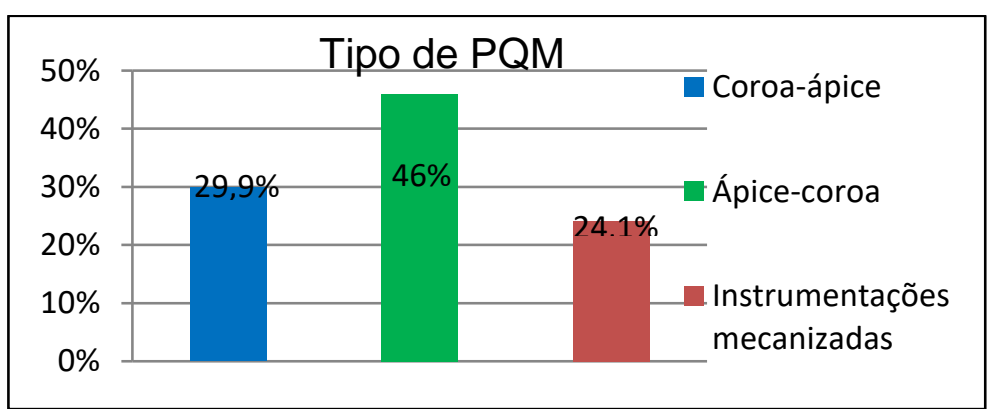

Figura 4 - Tipo de PQM que mais pode causar DPO. João

Pessoa/PB, 2016.

Como tratamento de alívio para a DPO ou dor trans-operatória, a maioria utiliza medicação sistêmica como coadjuvante, independentemente de ser polpa viva ou necrosada. Quanto ao tipo de medicação sistêmica utilizada por quadro clínico percebeu-se que (15,1\%) prescreveriam analgésicos e (11\%) antinflamatórios para um quadro de pulpite, $(6,8 \%)$ fariam associação medicamentosa para o flare up, o quadro de periodontite aguda seria tratado basicamente com 
antinflamatório $(7,8 \%)$ e antibiótico (10\%), já a periodontite crônica com antibioticoterapia (10\%) conforme o Figuras 5 e 6.

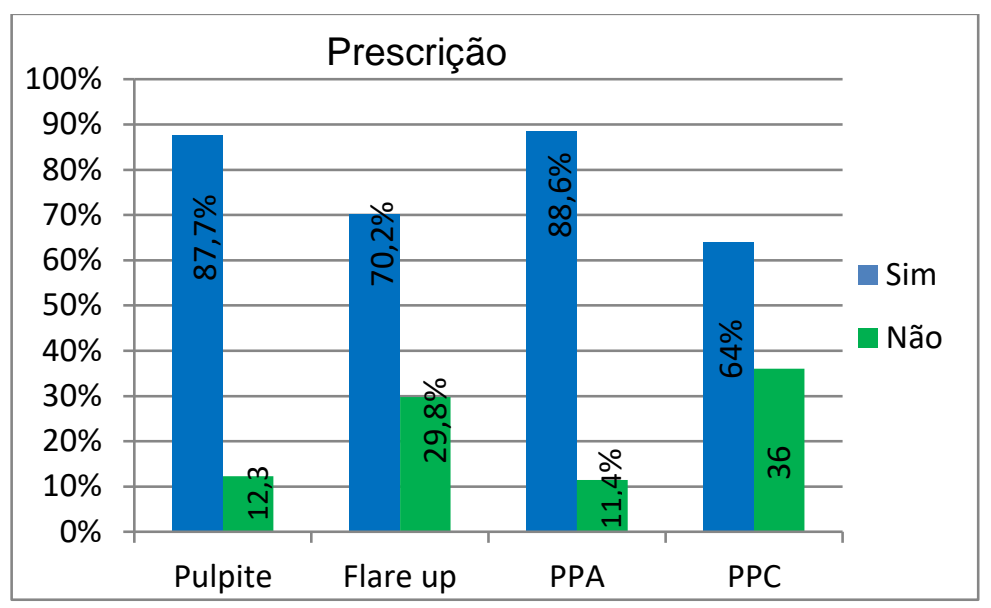

Figura 5 - Prescrição medicamentosa em casos de pulpite, flare up e patologias perirradiculares agudas e crônicas. João Pessoa/PB, 2016.

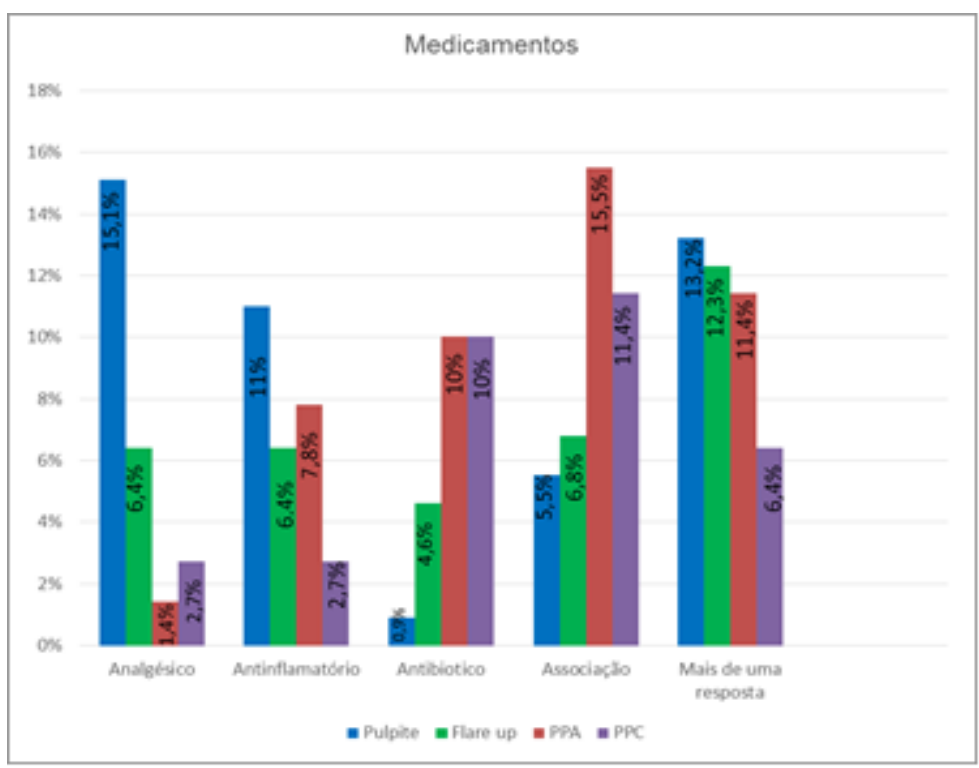

Figura 6 - Medicação de escolha em casos de pulpite, flare up. 


\section{Discussão}

Vários fatores têm sido associados a incidência de dor pós-operatória na endodontia, como quantidade de sessões (sessão única ou múltiplas sessões), substância irrigadora, técnica de preparo químico-mecânico utilizado no tratamento, sobreinstrumentação e sobreobturação, perfurações radiculares, tipo de medicação, sexo, idade, dente a ser tratado, entre outros. $\mathrm{Na}$ presente pesquisa foi avaliado o conhecimento dos acadêmicos acerca de dois fatores: tipo de substância química auxiliar e técnica de preparo químico-mecânico realizada ${ }^{7,8}$. A literatura aponta como uma das causas de dor pós operatória (DPO) pode ser a extrusão da quantidade excessiva da substância irrigadora pelo forame apical. E dentro dessas substâncias, a que mais provocaria uma DPO seria o hipoclorito em quantidade excessiva. Quando o hipoclorito de sódio é extruído além do canal radicular nos tecidos perirradiculares há uma queimadura química no local levando a necrose tecidual localizada ou extensiva. Uma reação inflamatória severa aguda dos tecidos perirradiculares se desenvolve, levando a edema e dor. Apesar de amplamente utilizado como substância química auxiliar, essa complicação não é tão comumente relatada? .

A respeito do tratamento ser em sessão única ou múltipla. Estudo mostrou que o tratamento em sessão única traz vantagens como redução da recontaminação e infiltração entre sessões, redução de custos e idas do paciente, prevenção da DPO e minimização de iatrogenias, no entanto para a escolha, deve ser levado em consideração fatores determinantes para o sucesso do tratamento, como por exemplo a habilidade profissional, condições de vitalidade do dente e presença de exsudato purulento ${ }^{10}$. Uma única sessão tem sido uma alternativa mais efetiva, especialmente em locais onde o paciente costuma faltar após a primeira consulta quando a dor foi atenuada ${ }^{11}$.

Ao longo dos anos, vários estudos sobre a compatibilidade biológica das soluções de hipoclorito de sódio têm sido realizados e mostraram que, quando em menores concentrações de 0,5\% e 1\%, as mesmas são bem toleradas pelos tecidos, no entanto, quando utilizadas a 5,25\% foi observada intensa toxicidade, resultado de alta injúria tecidual. Porém, as soluções com baixas concentrações como a solução de Dakin a 0,5\% apesar de ser bem biocompatível, apresenta baixo desempenho antimicrobiano, ficando a cargo de o profissional saber escolher a substância ideal para cada caso clínico ${ }^{12}$.

Uma das causas de DPO pode ser a extrusão da substância irrigadora pelo forame apical. Quando o hipoclorito de sódio é extruído além do canal radicular nos tecidos perirradiculares há uma queimadura química no local levando a necrose tecidual localizada ou extensiva. Uma reação 
inflamatória severa aguda dos tecidos perirradiculares se desenvolve, levando a edema e dor. Apesar de amplamente utilizado como substância química auxiliar, essa complicação não é tão comumente relatada ${ }^{9}$. A literatura aponta para uma maior incidência de DPO quando há extrusão de soluções mais concentradas de hipoclorito de sódio para os tecidos perirradiculares. A presente pesquisa, no entanto, mostrou que a maioria dos acadêmicos $(55,3 \%)$ acreditaram que a solução irrigadora não seria capaz de causar DPO e daqueles que acreditaram que solução poderia ser um fator causador de DPO, a maior parte marcou a solução de Milton como tendo uma maior capacidade de causar esse tipo de sintomatologia. Esse resultado não vai de encontro à maioria dos estudos uma vez que a solução de Milton apresenta concentração de $1 \%$, não sendo a mais citotóxica das soluções irrigantes, portanto, não sendo a principal causadora da dor pós-operatória.

Na maioria dos casos, autores indicam a solução de hipoclorito a 2,5\%. No entanto em casos de lesões periapicais o indicado seria em uma concentração maior, em torno de 5,25\% e no caso de polpa viva, uma concentração menor é mais indicado, sendo que esta concentração não poderia ser menor que $0,3 \%$ uma vez que não é eficaz contra alguns microrganismos como o Candida albicans e Estreptococus faecalis que fazem parte da microbiota dos canais radiculares ${ }^{13}$.

Na presente pesquisa averiguou-se que os alunos acreditaram que o tipo de Preparo Químico Mecânico (PQM) seria um fator causador da DPO (76,3\%), sendo a técnica ápice-coroa a que mais causaria DPO nos pacientes (46\%). Na atualidade a técnica mais utilizada é a coroa-ápice e uma ampla gama de instrumentos rotatórios vem surgindo no mercado odontológico. Poucos estudos comparam o tipo de PQM com a incidência e severidade da DPO. No entanto, a técnica ápice-coroa aumenta a chance de extrusão de detritos para os tecidos apicais, aumentando assim as chances de o paciente sentir DPO quando lança-se mão dessa técnica. Na tentativa de tratar os casos de dor trans e pós-operatória, a grande maioria dos alunos utilizaria a medicação sistêmica como coadjuvante no tratamento endodôntico independente de ser um quadro de polpa viva ou necrosada ${ }^{14}$.

Atualmente, o mercado tem lançado cada vez mais tecnologias às técnicas mecanizadas que são bastante utilizadas devido a redução do tempo clínico em relação à técnica manual. Um estudo afirma que em relação a extrusão de detritos para os tecidos periapicais é menor na técnica mecanizada do que na técnica manual, uma vez que os instrumentos mecanizados levam as raspas de dentina para a porção cervical da raiz, sendo facilmente removidas pela irrigação ${ }^{15}$.Isso permite concluir uma das vantagens desses instrumentos em relação a DPO.

De acordo com a literatura vigente em um quadro de pulpite havendo a intervenção endodôntica, não haveria necessidade de medicação sistêmica, no entanto, alguns autores indicam 
a prescrição de analgésico e anti-inflamatório para evitar um quadro de DPO ${ }^{14}$. Além disso, a periodontite apical aguda é caracterizada pela presença de processo inflamatório agudo, sem a formação franca de um abscesso. Para este quadro recomenda-se prescrição de analgésico e antiinflamatório para combater a DPO, no entanto, não seria necessário uso de antibióticos. No entanto, $5,5 \%$ dos alunos prescreveriam uma associação de medicamentos e 0,9\% prescreveriam antibiótico para pulpite, agindo de forma errônea. Poucos alunos (10\%) avaliados prescreveriam antibióticos e 15,5\% uma associação medicamentosa na situação de periodontite apical aguda.

A resistência microbiana emergiu como um dos principais problemas de saúde pública. Doenças que no passado eram tratadas com eficiência por meio da antibioticoterapia agora requerem o uso de outro agente antimicrobiano, em geral, mais caro e mais tóxico e que ainda assim pode ser ineficaz. Daí a importância do conhecimento por parte de alunos e profissionais com relação ao uso de medicação sistêmica na endodontia, que é uma área da odontologia ligada muitas vezes à dor, mas que a intervenção endodôntica é mais importante no tratamento dessa dor do que a medicação sistêmica ${ }^{14}$.

Por fim esse tipo de estudo realizado através de questionários provê informações a respeito do conhecimento, atitudes, preferências, opiniões, experiências e práticas dos acadêmicos em formação. No entanto, pode apresentar dificuldades, sendo a principal delas a recusa em participar, gerando um índice pequeno de participantes, o que reduz a representatividade da pesquisa. Resultados gerados podem ajudar a nortear o ensino da endodontia ainda na graduação com a finalidade de reduzir ao máximo o índice de acidentes e complicações no decorrer do tratamento.

\section{Conclusão}

Concluiu-se com a presente pesquisa que os alunos do último ano do curso de odontologia apresentam conhecimento limitado a respeito da dor pós-operatória e no critério substância química auxiliar, e com relação ao uso de medicação sistêmica, há uma preocupação maior, pois uma boa parte dos alunos faria uso da prescrição medicamentosa sem necessidade. Portanto, os dados da pesquisa apontaram para necessidade de mudanças nas metodologias de ensino nas disciplinas de farmacologia e terapêutica medicamentosa, propondo-se uma maior integração das mesmas às práticas clínicas. 


\section{Referências}

1. Al Zaka IM, The Incidence of Pain after Root Canal Treatment Using Different Irrigation Methods. Tikrit Journal for Dental Sciences, 2012;1(1):38-43

2. Martin-Gonzalez J, Echevarria-Perez M, Sanchez-Dominguez B, Tarilonte-Delgado ML, Castellanos-Cosano L, Lopez-Frias FJ, et al. Influence of root canal instrumentation and obturation techniques on intra-operative pain during endodontic therapy. Med Oral Patol Oral Cir Bucal. 2012;17(5):912-918.

3. Harrison JW, Baumgartner JC, Svec TA. Incidence of pain associated with clinical factors during and after root canal therapy. Part 1. Interappointment pain. J Endod. 1983;9(9):384-387.

4. Almeida G, Marques E, De Martin AS, da Silveira Bueno CE, Nowakowski A, Cunha RS. Influence of irrigating solution on postoperative pain following single-visit endodontic treatment: randomized clinical trial. J Can Dent Assoc. 2012;78:c84.

5. Cunningham CJ, Mullaney TP. Pain control in endodontics. Dent Clin North Am. 1992;36(2):393-408.

6. Andrade ED; Souza Filho, FJ. Terapêutica medicamentosa em odontologia. 2. ed. São Paulo: Artes Médicas, 2006.

7. Estrela C, Moraes TA, Brugnera JA, Almeida DR, Djalma PJ. Dor pós-operatória em dentes com inflamação pulpar - revisão sistemática. ROBRAC. 2006;15(40):1-12.

8. Ng YL, Glennon JP, Setchell DJ, Gulabivala K. Prevalence of and factors affecting postobturation pain in patients undergoing root canal treatment. International Endodontic Journal. 2004;37(6):381-391.

9. Spencer HR, Ike V, Brennan $P$ a. Review: the use of sodium hypochlorite in endodontics-potential complications and their management. Br Dent J. 2007;202(9):555-559.

10. Endo $\mathrm{M}$ et al. Endodontia em sessão única ou múltipla: revisão de literatura. RFO. 2015;20(3):408-413.

11. Estrela,C et al. Dor pós-operatória em dentes com infeções. RGO. 2008;56(4):353-359.

12. Ribeiro ECC et al. O Hipoclorito de Sódio na Endodontia. Brazilian Journal of Health. 2010 jan;1(1):54-62.

13. Borin G, Becker AN, Oliveira EPM de. A história do hipoclorito de sódio e a sua importância como substância auxiliar no preparo químico mecânico de canais radiculares. Rev Endodontia Pesqui e Ensino. 2007;3(5):1-5.

14. Martins ELR de S, Torino GG, Martins GB. Antibióticos em endodontia: por que, como e quando usá-los?. Rev Bras Odontol. 2014.

15. Semaan FS, Fagundes FS, Haragushiku G, Leonardi DP, Baratto Filho F. Endodontia mecanizada: a evolução dos sistemas rotatórios contínuos. RSBO. 2009;6(3):297-309. 